\title{
Anti-thyroidal peroxidase antibodies are associated with thyrotropin levels in hypothyroid patients and in euthyroid individuals
}

\author{
Barbara Bromińska ${ }^{1 *}$, Gabriel Bromiński ${ }^{{ }^{*}}$, Maciej Owecki ${ }^{1}$, Michał Michalak², \\ Agata Czarnywojtek', Ryszard Waśko', Marek Ruchała' \\ ${ }^{1}$ Department of Endocrinology, Metabolism and Internal Medicine, University of Medical Sciences, Poznań, Poland \\ 2 Department of Informatics and Statistics, University of Medical Sciences, Poznań, Poland
}

* Authors who contributed equally to the study.

Bromiński G, Bromińska B, Owecki M, Michalak M, Czarnywojtek A, Waśko R, Ruchała M. Anti-thyroidal peroxidase antibodies are associated with thyrotropin levels in hypothyroid patients and in euthyroid individuals. Ann Agrice Environ Med. $2017 ; 24(3)$ : $431-434$. doi: $10.5604 / 12321966.1232090$

\begin{abstract}
Objective. The study was designed to evaluate the relationship between thyroid antibodies and gland dysfunction, with the aim of finding a clinically useful threshold value of thyreoperoxidase antibodies, which could prove to be predictive for thyroid failure.

Materials and method. The study was conducted on 99 women, ages ranging from 18-91 years (mean age: $45.5 \pm 17.0$ ), were treated as outpatients in the Department of Endocrinology, Metabolism and Internal Medicine. Analysis of serum samples for TSH concentration and anti-TPO titers was conducted.

Results. The most common disorder was hypothyroidism. Anti-TPO titers above reference range values were observed in 35 patients (35.4\%): 21 (60\%) were hypothyroid and 11 (31.4\%) were euthyroid. The anti-TPO and TSH serum levels correlated both in patients with high thyroid antibody titers, and in the anti-TPO negative groups. To find the threshold value of antiTPO that would help predict hypothyroidism, receiver operating curves were used. With this approach, TPO antibody titers over $17 \mathrm{IU} / \mathrm{ml}$ indicated hypothyroidism with a $90 \%$ sensitivity and $75 \%$ sensibility.

Conclusion. It can be postulated that the cutoff values of anti-TPO in the general population should be decreased in order to improve autoimmune thyroid disorder screening. Obviously, using that margin may lead initially to the detection of some false positive subjects. However, with lower cut-off values, more patients can be enrolled into thyroid follow-up groups. In this way, many people could avoid complications of undiagnosed, insidious thyroid failure.
\end{abstract}

- Key words

anti-TPO, autoimmunity, hypothyroidism, euthyroidism, TSH, thyreoperoxidase

\section{INTRODUCTION}

Thyreoperoxidase (TPO) is a membrane-bound glycoproteinaceous enzyme which plays a crucial role in thyroid hormone synthesis. Iodination and successive coupling of tyrosine residues catalysed by TPO, finally results in T3 and T4 production [1]

Inadequate immunological response against TPO triggers the onset of autoimmune thyroid disease (ATD). Not only does anti-TPO induce antibody-mediated cytotoxicity, but it also contributes to complement cascade activation, and is probably able to directly inhibit TPO activity. Consequently, thyroid cell damage, as well as direct enzyme blocking, may lead to insufficient hormone production, occasionally preceded by transient hyperthyroidism [2]. Both hypo- and hyperthyroidism are among the most common endocrine disorders $[3,4,5]$. In addition, elevated titers of anti-TPO were revealed in a wide range of diseases affecting various body organs, including Biermer's pernicious anaemia, viral hepatitis treated with INFa, systemic lupus erythematosus,

Address for correspondence: Barbara Bromińska, Department of Endocrinology, Metabolism and Internal Medicine, University of Medical Sciences in Poznań, Poland E-mail:gabriel.brominski@gmail.com

Received: 17 Octomber 2013; accepted: 23 May 2014; first published: January 2017 insulin-dependent diabetes mellitus, breast cancer, vitiligo [6] and thyroid nodular disease [7, 8]. Moreover, relationships between anti-TPO and obstetric complications, such as postpartum thyroiditis [9] or depression after labour, are well known [10].

In spite of the information presented above, the detection of TPO antibodies may sometimes create confusion: although detecting markers of immunological response acts as a hallmark for diagnosis of ATD on the one hand [11], on the other, anti-TPO may be discovered in $10 \%$ of the population without overt thyroid impairment [12]. In addition, the impact of increased anti-TPO titers on future thyroid dysfunction also remains controversial. Some studies have shown that the presence of anti-TPO in the bloodstream is related to an increased risk of thyroid failure in future life [13]. Nevertheless, TPO antibodies are elevated in some euthyroid individuals who will never demonstrate insufficient hormone production [14]. Therefore, the question of the relationship between anti-TPO and thyroid dysfunction remains unclear, and under these circumstances, finding the exact level of anti-TPO that is diagnostic for any particular thyroid disease would shed some light on the problem. This approach was used in the presented study: as the prevalence of TPO antibodies may vary in different types 
of thyroid diseases, it would be of interest to investigate the complicated relationship between autoimmunity and fragile thyroid hormone balance. In this way, one could observe the associations between the presence of autoimmunity and the type of thyroid disorder. In addition, it would be clinically useful to try to find some cutoff value for antiTPO levels that would be characteristic for a given thyroid disease. Therefore, the presence of anti-TPO was examined in a population of patients with different hormonal thyroid status, and the relationships between anti-TPO and thyroid function investigated.

\section{MATERIALS AND METHOD}

The patients were being treated in an Endocrine Outpatient Clinic for various thyroid disorders. The study group comprised 99 women, ages ranging from 18-91 years (mean age: $45.5 \pm 17.0)$. In the morning after an overnight fast, venous blood samples were collected from all participants and evaluated for anti-thyreoperoxidase antibodies and TSH serum concentration. Both anti-TPO titers and TSH levels were assessed with electrochemiluminescence immunoassay (ECLIA). The former with sensitivity of $0.005 \mathrm{uIU} / \mathrm{mL}$, the later - $5 \mathrm{IU} / \mathrm{mL}$ (Roche TSH III gen. and Roche Anti-TPO; analyzer Roche Cobas e 601, production 2009).

The patients were classified according to anti-TPO serum levels. Based on the reference range, they were divided into anti-TPO positive $(\geq 34 \mathrm{IU} / \mathrm{ml})$ and anti-TPO negative $(<34 \mathrm{IU} / \mathrm{ml})$ groups. Moreover, according to the thyroid function, the patients were also divided into hypo-, hyper- and euthyroid subjects. Participants who received L-thyroxin were assigned to the group with insufficient hormone production, while those treated with methimazole were classified as hyperthyroid. Further, in order to assess correlations between anti-TPO serum titers and TSH level, patients on medication were set aside, thereby creating a group of 73 people. This excluded the impact of L-thyroxin and methimazole treatment on TSH levels that would otherwise influence the results.

\section{RESULTS}

In the entire study group, the mean TSH serum level was $2.95 \mathrm{IU} / \mathrm{ml}$ (SD \pm 3.79 ), varying from $0.01-24.92$. There were 30 hypothyroid patients (TSH levels: 0.97-24.92 IU/ml), 9 with thyroid hormone overproduction (TSH levels: $0.01-$ $4.47 \mathrm{IU} / \mathrm{ml}$ ), and 60 were classified as being euthyroid (TSH levels: $0.56-3.95 \mathrm{IU} / \mathrm{ml}$ ). Anti-TPO assay showed values above the threshold in 35 subjects (35.4\%). Those markers of immunological response were significantly more prevalent in hypothyroid individuals than in those who were euthyroid $(\mathrm{p}=0.00001)$ (Fig. 1). It is essential to note that in the antiTPO positive patients, the most common thyroid disorder was hypothyroidism - 21 subjects $(60 \%)$, while 3 (8.6\%) were hyperthyroid. On the other hand, up to 11 patients (31.4\%) in that subdivision were euthyroid. Furthermore, individuals without elevated antibody titers were most frequently reported to have normal thyroid function - 49 subjects (76.6\%), whereas 9 participants (14.1\%) displayed impaired hormone production. Hyperthyroidism was disclosed in 6 individuals $(9.4 \%)(\mathrm{p}=0.00001)$.

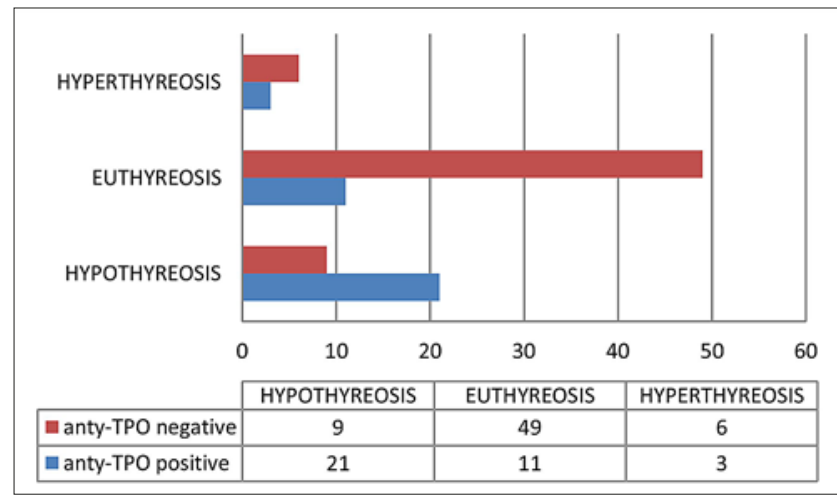

Figure 1. Anti-TPO and thyroid hormonal status

Analysis which encompassed 73 subjects, concerning the relationship between TSH and anti-TPO serum level, was conducted. A significant correlation was observed in the entire examined group, as well as in the anti-TPO positive group. In addition, a similar connection was also found in the negative subdivisions (Fig. 2, 3) $(\mathrm{p}=0)$.

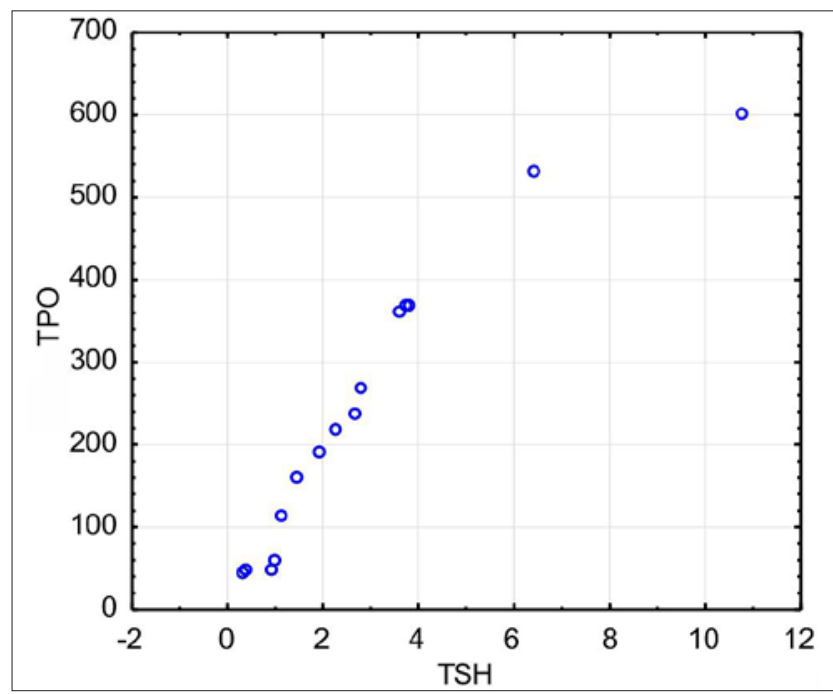

Figure 2. Correlation between TSH serum levels and anti-TPO titers in anti-TPO positive group

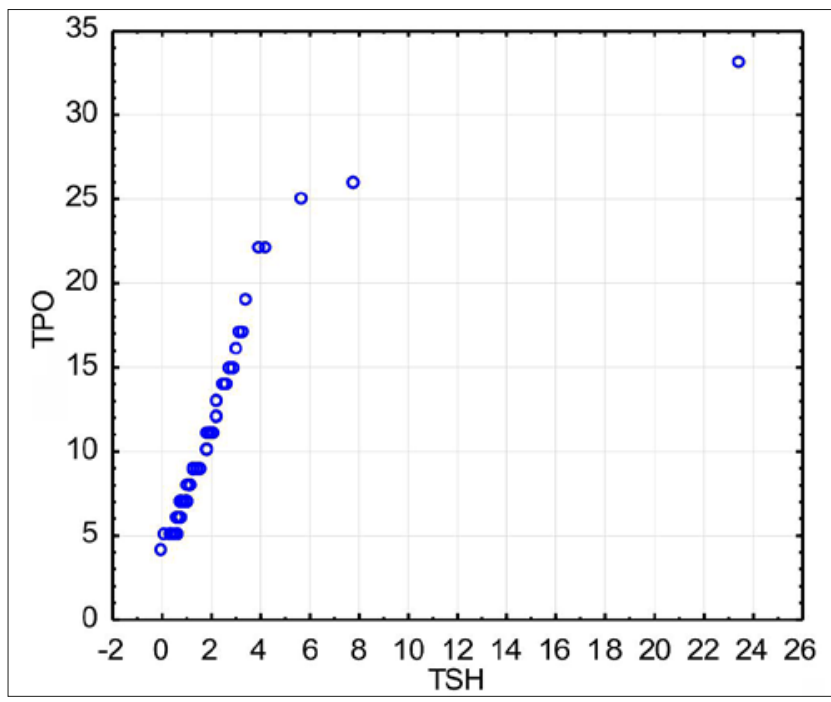

Figure 3. Correlation between TSH serum levels and anti-TPO titers in anti-TPO negative group 
Finally, the measurement of TPO antibodies in the bloodstream was found to be a useful tool for predicting scanty hormone production. A proposed threshold value of anti-TPO titers over $17 \mathrm{IU} / \mathrm{ml}$ indicated hypothyroidism with a $90 \%$ sensitivity and $75 \%$ sensibility (Fig. 4).

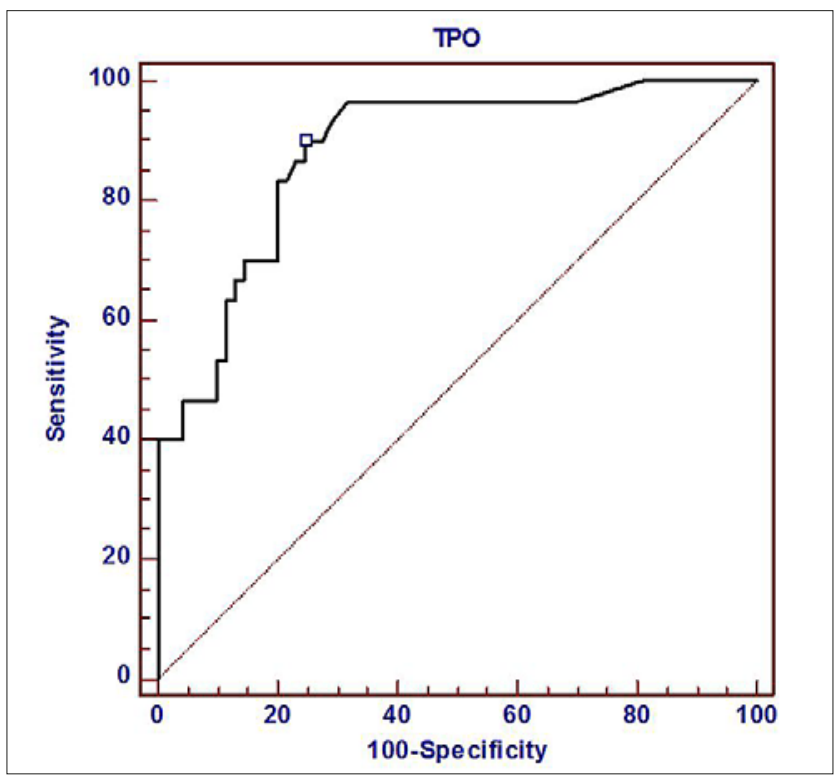

Figure 4. Sensitivity and specificity of serum TPO measurement

Conversely, the TSH serum concentration could not serve to prognosticate the occurrence of TPO antibodies. The best chosen threshold for TSH of $2.6 \mathrm{IU} / \mathrm{ml}$ was associated with only $43.75 \%$ sensitivity and $80.7 \%$ specificity. When the cutoff value was lower, more false positive results emerged, whereas with an increased TSH threshold, the opposite effect was observed.

\section{DISCUSSION}

As shown by the presented study, the major thyroid disorder was hypothyroidism, whereas exaggerated hormone excretion occurred rarely. This notion was not surprising because Poland is an iodine-replete intake country [15]. In such regions, thyroid failure occurs more frequently than thyrotoxicosis, which dominates in iodine-deficient areas [16]. Despite the fact that medical staff are well-trained in the recognition and treatment of thyroid impairment, the rates of overlooked disorders are still high. Referring to previous studies, formerly undiagnosed overt hypothyroidism ranged from $0-7 / 1000$ in male subjects, and from 3.3-20.5/1000 female subjects [17]. Therefore, due to severe side effects of thyroid impairment $[18,19]$, a tool for predicting such abnormalities would be useful.

In iodine sufficient areas, acquired inadequate hormone excretion develops mostly due to an autoimmune reaction or treatment for thyrotoxicosis [20]. Own research revealed that $70 \%$ of hypothyroid patients were anti-TPO positive, which strongly suggested an autoimmune background. Based on that data, the role of anti-TPO was evaluated in relation with gland function. Obviously, to avoid the influence of treatment on TSH levels, patients who received any thyroid medication were excluded. With this approach, the natural relationships between anti-TPO levels and TSH concentrations could be observed. This analysis disclosed a significant correlation between TSH levels and anti-TPO titers in the group composed of individuals without medication for thyroid disorders. Surprisingly, this connection was present in either negative or positive subdivision $(\mathrm{R}=1 ; \mathrm{p}<0.05)$. It was therefore concluded that even within blood samples in which antibody llevels were under the cutoff value, the increase of their titers is connected with the rise in TSH.

The novelty of the current study is that other investigators have shown similar outcomes, but only in populations with elevated anti-TPO in the bloodstream. Besides, they revealed that not only hypo- but also hyperthyroidism was associated with positive anti-TPO [21]. This may point to the possibility of determining an anti-TPO threshold for predicting hypothyroidism. Consequently, in the current study, mathematical analyses were performed to find a new threshold for anti-TPO that would be indicative of hypothyroidism, and conversely, a TSH threshold that would be specific for antiTPO increase. This analysis determined a cutoff value for anti-TPO titers of over $17 \mathrm{IU} / \mathrm{ml}$, which indicated insufficient hormone production with $90 \%$ sensitivity and $75 \%$ specificity. Obviously, one should be aware that using that margin leads to the detection of some false positive subjects, although the sensitivity is satisfactory. This data could be supported by the results research by Whickham, in which both elevated serum TSH and TPO antibodies levels, separately or together, were presented as a risk factor for hypothyroidism [22]. Moreover, referring to Prummel, accidental disclosure of elevated antiTPO serum titers should obligate us to evaluate thyroid function. Anti-TPO antibodies evaluation can be performed as a first laboratory test in the case of the presence of other autoimmune diseases in relatives of patients with ATD, prone pregnant women, subjects on specific drug therapy, as well as women planning gestation with certain risk factors for autoimmune disorders [14]. These are valuable clinical implications.

Although the detection of anti-TPO proves to be useful in predicting hypothyroidism, the significance of the markers of immunological response remains undetermined.

Recent cross-sectional studies have demonstrated a high prevalence of those antibodies within the general population. In Holland, such globulins were shown in $8.6 \%$ of males and in $18.5 \%$ of females, respectively [23]. In the presented study, higher rates of elevated titers of anti-TPO antibodies (35.4\% participants, all women) were discovered, presumably due to the character of the studied group. Besides, it was demonstrated that $11.1 \%$ euthyroid participants were antiTPO positive. It is known that individuals without any thyroid disorder, as well as patients, who are afflicted with a wide range of disorders, may reveal a presence or elevation in anti-TPO antibody titres. However, in the 20 -year follow-up to Whickham's study, it was disclosed that the annual risk for apparent impaired gland function was $2 \%$ for a women with raised antithyroidal antibodies. Not every subject become hypothyroid after observation, a few even eliminated markers of immunological response from their bloodstream.

Finally, in the current study, approximately one-third of the anti-TPO positive patients were euthyroid. This phenomenon could be explained by the thesis that antithyroidal antibodies are heterogeneous; thus their presence does not always indicate impelling thyroid failure [24]. The other possible illustration for the presence of markers of immunological response in healthy subjects has been described by Zöphel 
et al. When the measurement of anti-TPO was conducted by chemiluminescence assay, a highly sensitive method, the authors showed that the euthyroid population followed Gaussian distribution for those antibodies under very low values, namely $1.1 \mathrm{IU} / \mathrm{ml}$. Antithyroidal antibodies were detected in sera of almost every euthyroid participant [25], which may suggest that anti-TPO are commonly present in human serum.

\section{CONCLUSIONS}

The presented study shows that the relationship between thyroid markers of immunological response and its function remains unsettled. Although thyroid antibodies act as a hallmark for autoimmune reactions, euthyroid subjects may either disclose their presence, or adversely, even within the anti-TPO negative group, autoantibodies were positively correlated with TSH concentration. In spite of this, the aim was to define a new lower cutoff value for anti-TPO in order to predict hypothyroidism. This proposed new threshold value may help distinguish afflicted patients from the healthy subjects, but certainly cannot serve to establish a definitive diagnosis. Instead, it may indicate a need for further clinical evaluation in a given individual.

\section{Conflict of interests}

The authors declare that no competing financial interests exist between them.

\section{REFERENCES}

1. Weetman A P.Thyroid peroxidase as antigen in autoimmune thyroiditis. Clin Exp Immunol. 1990; 80(1): 1-3. 2. Ruf J, Carayon P. Structural and functional aspects of thyroid peroxidase. Arch Biochem Biophys. 2006; 445(2): 269-277.

2. Bjoro T, Holmen J, Krüger O, Midthjell K, Hunstad K, Schreiner T et al. Prevalence of thyroid disease, thyroid dysfunction and thyroid peroxidase antibodies in a large, unselected population. The Health Study of Nord-Trùndelag (HUNT). Eur J Endocrinol 2000; 143(5): 639-647.

3. Dhanwal DK. Thyroid disorders and bone mineral metabolism. Indian J Endocrinol Metab. 2011; 15(Suppl 2): S107-112.

4. Kostoglou-Athanassiou I, Ntalles K. Hypothyroidism - new aspects of an old disease. Hippokratia. 2010; 14(2):82-87.

5. Estienne V, Duthoit C, Costanzo VD, Lejeune PJ, Rotondi M, Kornfeld $\mathrm{S}$ et al. Multicenter study on TGPO autoantibody prevalence invarious thyroid and non-thyroid diseases; relationships with thyroglobulin and thyroperoxidase autoantibody parameters. Eur J Endocrinol. 1999; 141(6): 563-569.

6. Bülow Pedersen I, Laurberg P, Knudsen N, Jørgensen T, Perrild H, Ovesen L et al. A population study of the association between thyroid autoantibodies in serum and abnormalities in thyroid function and structure. Clin Endocrinol (Oxf). 2005; 62(6): 713-720.
7. Gabryel B, Brominski G, Owecki M, Michalak M, Ruchala M. The prevalence of thyroid nodular disease in patients with increased titers of anti-thyroidal peroxidase antibodies. Neuro Endocrinol Lett. 2012; 33(4): 442-445.

8. Stagnaro-Green A. Postpartum thyroiditis. Best. Pract. Res. Clin. Endocrynol. Metab. 2004; 18(2): 303-316

9. Ruchała M, Zybek A, Bromińska B, Szczepanek-Parulska E. Thyroid autoimmunity in pregnancy- a problem of mother and child. Arch Perinat Med. 2012; 18(2): 86-91.

10. Prummel MF, Strieder T, Wiersinga WM. The environment and autoimmune thyroid diseases. Eur J Endocrinol. 2004; 150(5): 605-618.

11. Ajjan RA, Weetman AP. Thyroid Autoantibody Measurement. In: Brent GA (ed.) Thyroid Function Testing, Nowy Jork 2010 p. 109-124.

12. O'Leary PC, Feddema PH, Michelangeli VP, Leedman PJ, Chew GT, Knuiman $\mathrm{M}$ et al. Investigations of thyroid hormones and antibodies based on a community health survey: the Busselton thyroid study. Clin Endocrinol (Oxf). 2006; 64(1): 97-104.

13. Prummel MF, Wiersinga WM. Thyroid peroxidase autoantibodies in euthyroid subjects. Best Pract Res Clin Endocrinol Metab. 2005; 19(1): 1-15.

14. Szybiński Z, Jarosz M, Hubalewska-Dydejczyk A, Stolarz-Skrzypek $\mathrm{K}$, Kawecka-Jaszcz K, Traczyk I et al. Iodine-deficiency prophylaxis and the restriction of salt consumption - a 21st century challenge. Endokrynol Pol. 2010; 61(1): 135-140.

15. Laurberg P, Pedersen KM, Hreidarsson A, Sigfusson N, Iversen E, Knudsen PR. Iodine intake and the pattern of thyroid disorders: a comparative epidemiological study of thyroid abnormalities in the elderly in Iceland and in Jutland, Denmark. J Clin Endocrinol Metab. 1998; 83(3): 765-769.

16. Vanderpump MP. Epidemiology of Thyroid Dysfunction Hypothyroidism and Hyperthyroidism. Thyroid international. 2009; 2: 1-14.

17. Kim M, Ladenson P. Thyroid. In: Goldman L, Schafer AI (eds.) Cecil Medicine, Philadelphia 2011 chapter 233.

18. Ruchala M, Kosowicz J, Baumann-Antczak A, Skiba A, Zamyslowska $\mathrm{H}$, Sowinski J. The prevalence of autoantibodies to: myosin, troponin, tropomyosin and myoglobin in patients with circulating triiodothyronine and thyroxine autoantibodies (THAA). Neuro Endocrinol Lett. 2007; 28(3): 259-266.

19. Vanderpump MP, Tunbridge WM. Epidemiology and Prevention of Clinical and Subclinical Hypothyroidism. Thyroid. 2002; 12(10): 839-847.

20. Hollowell JG, Staehling NW, Flanders WD, Hannon WH, Gunter EW, Spencer CA et al. Serum TSH, T4, and Thyroid Antibodies in the United States Population (1988 to 1994): National Health and Nutrition Examination Survey (NHANES III). J Clin Endocrinol Metab. 2002; 87(2): 489-499.

21. Vanderpump MP. The epidemiology of thyroid disease. Br Med Bull. 2011; 99: 39-51.

22. Hoogendoorn EH, Hermus AR, de Vegt F, Ross HA, Verbeek AL, Kiemeney LA et al. Thyroid function and prevalence of antithyroperoxidase antibodies in a population with borderline sufficient iodine intake: influences of age and sex. Clin Chem. 2006; 52(1): 104-111.

23. Kaczur V, Vereb G, Molnár I,, Krajczár G, Kiss E, Farid NR et al. Effect of anti-thyroid peroxidase (TPO) antibodies on TPO activity measured by chemiluminescence assay. Clin Chem. 1997; 43(8 Pt 1): 1392-1396.

24. Zöphel K, Saller B, Wunderlich G, Grüning T, Koch R, Wilde J et al. Autoantibodies to thyroperoxidase (TPOAb) in a large population of euthyroid subjects: implications for the definition of TPOAb reference intervals. Clin Lab. 2003; 49(11-12): 591-600. 